\title{
Fueter regular mappings and harmonicity
}

\author{
by WiESŁAW KRÓLIKOWSKI (Łódź)
}

\begin{abstract}
It is shown that Fueter regular functions appear in connection with the Eells condition for harmonicity. New conditions for mappings from 4-dimensional conformally flat manifolds to be harmonic are obtained.
\end{abstract}

1. Introduction. There does not exist a "quaternionic analysis" in the same sense as complex analysis. This term stands for a theory investigating properties of regular functions in the sense of Fueter. Let us recall their definition.

Definition 1.1. A function $f: \Omega \rightarrow \mathbb{H}$ ( $\mathbb{H}$ the quaternions) is said to be regular in a domain $\Omega \subset \mathbb{H}$ if $f$ is differentiable in the usual sense as a mapping of $\Omega$, considered as an open set in $\mathbb{R}^{4}$, with values in $\mathbb{R}^{4}$ and

$$
D^{+} f:=\frac{1}{4}\left(\partial_{0}+i_{1} \partial_{1}+i_{2} \partial_{2}+i_{3} \partial_{3}\right)\left(f_{0}+i_{1} f_{1}+i_{2} f_{2}+i_{3} f_{3}\right)=0
$$

in $\Omega$, where $i_{1}, i_{2}, i_{3}$ are the quaternionic units.

These functions are the most natural analog of holomorphic functions. But because of the non-commutativity of quaternions one cannot define a quaternionic manifold with Fueter regular functions as transition functions.

Nevertheless, one does define a "quaternionic manifold" omitting completely the problem of transition functions. This definition is connected with the so-called "Berger list" [1]. Berger proved that the holonomy group of every irreducible Riemannian manifold which is not a symmetric space is a subgroup of one of the following:

$$
\begin{gathered}
S O(n), \quad U(n / 2), \quad \operatorname{Sp}(n / 4) \times \operatorname{Sp}(1), \quad \operatorname{Sp}(n / 4), \\
G_{2}(n=7), \quad \operatorname{Spin}(7)(n=8), \quad \operatorname{Spin}(9)(n=16),
\end{gathered}
$$

where $n$ denotes the dimension of the manifold in question.

1991 Mathematics Subject Classification: 53C10, 53C40, 58E20.

Key words and phrases: quaternions, Fueter regular functions, harmonic mappings. 
The manifolds whose holonomy group is contained in $S O(n)$ are oriented Riemannian manifolds. One can prove only general theorems about the topology of such manifolds.

The manifolds with holonomy group in $U(n / 2)$ are complex Kähler manifolds. They play a very important role in complex analysis.

The next group from the Berger list constitutes a basis of the following definition:

Definition 1.2. A 4n-dimensional Riemannian manifold is called quaternionic (precisely, quaternionic-Kähler) if its holonomy group is a subgroup of $\operatorname{Sp}(n) \times \operatorname{Sp}(1)$ (see e.g. [2]).

Analysis on such manifolds is another kind of "quaternionic analysis". At first sight it has nothing to do with Fueter regular functions.

In this paper it is shown that Fueter regular functions do appear on quaternionic manifolds. It turned out, to our surprise, that the Eells condition for harmonicity [5] is, for some kind of 4-dimensional manifolds, equivalent to the existence of an antiregular function in the sense of Fueter. It is the most significant result of the present paper. This once more points to the importance of Fueter regular functions. In particular, rewriting the Eells condition for harmonicity in quaternionic variables we obtain new results on harmonic mappings from 4-dimensional conformally flat manifolds.

In particular, we get a very interesting new characterization of harmonic mappings from the 4-dimensional torus.

Some of these problems were discussed at the University "La Sapienza" in Rome. The author wants to express his hearty thanks to Prof. Stefano Marchiafava for numerous discussions and encouragement in this work.

The manifolds considered in the paper are assumed to be connected, compact, orientable and without boundary.

2. Elements of quaternionic analysis. Denote by $\mathbb{H}$ the field of quaternions. Let $V$ be a real $m$-dimensional vector space and $V^{*}$ the dual space to $V$. Consider the following vector spaces over $\mathbb{H}$ :

$$
\begin{aligned}
V^{\mathbb{H}}:= & V \otimes_{\mathbb{R}} \mathbb{H}-\text { quaternionification of } V \text { (a right } \mathbb{H} \text {-vector space), } \\
\overline{V^{\mathbb{H}}:=} & \mathbb{H} \otimes_{\mathbb{R}} V \text { - quaternionic conjugation of } V \text { (a left } \mathbb{H} \text {-vector space), } \\
& \left(V^{\mathbb{H}}\right)^{*}:=\mathbb{H} \otimes_{\mathbb{R}} V^{*} \text { - dual of } V^{\mathbb{H}} \text { (a left } \mathbb{H} \text {-vector space), } \\
& \left(\overline{V^{\mathbb{H}}}\right)^{*}:=V^{*} \otimes_{\mathbb{R}} \mathbb{H} \text { - dual of } \overline{V^{\mathbb{H}}} \text { (a right } \mathbb{H} \text {-vector space). }
\end{aligned}
$$

We use the fact that $\overline{q v}=\overline{v q}, v \in V^{\mathbb{H}}, q \in \mathbb{H}$.

Let $(1, i, j, k)$ be a fixed standard basis of $\mathrm{H}$ with the well known properties: $i^{2}=j^{2}=k^{2}=-1, i j k=-1$. Consider three involutions of $\mathbb{H}$, denoted by $\sigma_{1}, \sigma_{2}$ and $\sigma_{3}$, given by automorphisms corresponding to $i, j, k$, 
respectively, which satisfy the following conditions:

$$
\begin{array}{ll}
\sigma_{1}^{2}=\sigma_{2}^{2}=\sigma_{3}^{2}=\mathrm{id}, & \sigma_{1} \sigma_{2}=\sigma_{2} \sigma_{1}=\sigma_{3}, \\
\sigma_{2} \sigma_{3}=\sigma_{3} \sigma_{2}=\sigma_{1}, & \sigma_{3} \sigma_{1}=\sigma_{1} \sigma_{3}=\sigma_{2} .
\end{array}
$$

Using quaternionic units we can write

$$
\sigma_{1}(q)=-i q i, \quad \sigma_{2}(q)=-j q j, \quad \sigma_{3}(q)=-k q k, \quad q \in \mathbb{H} .
$$

Set

$$
q^{1}:=\sigma_{1}(q), \quad q^{2}:=\sigma_{2}(q), \quad q^{3}:=\sigma_{3}(q), \quad q \in \mathbb{H} .
$$

Explicitly, if $q=x_{0}+i x_{1}+j x_{2}+k x_{3}$, then

$$
\begin{aligned}
& q^{1}=x_{0}+i x_{1}-j x_{2}-k x_{3}, \\
& q^{2}=x_{0}-i x_{1}+j x_{2}-k x_{3}, \\
& q^{3}=x_{0}-i x_{1}-j x_{2}+k x_{3} .
\end{aligned}
$$

Right multiplication by $i, j$ and $k$ determines a triple $\left(I_{1}, I_{2}, I_{3}\right)$ of complex structures on $\mathbb{R}^{4 m} \cong \mathbb{H}^{m}$ satisfying the following conditions:

$$
I_{1}^{2}=I_{2}^{2}=I_{3}^{2}=-\mathrm{Id}, \quad I_{1} I_{2} I_{3}=-\mathrm{Id},
$$

where Id stands for the identity mapping of $\mathbb{R}^{4 m}$.

Any two such triples $\left(I_{1}, I_{2}, I_{3}\right)$ and $\left(I_{1}^{\prime}, I_{2}^{\prime}, I_{3}^{\prime}\right)$ are related by a transformation

$$
I_{h}^{\prime}=\sum_{k=1}^{3} c_{h k} I_{k}, \quad h=1,2,3
$$

with $\left(c_{h k}\right) \in S O(3)$.

Let $(M, g)$ be a Riemannian manifold.

Definition 2.1 [2]. An almost quaternionic structure is defined as a covering $\left\{U_{i}\right\}$ of $M$ with two almost complex structures $I_{i}$ and $J_{i}$ on each $U_{i}$ such that $I_{i} J_{i}=-J_{i} I_{i}$ and the 3 -dimensional vector spaces of endomorphisms generated by $I_{i}, J_{i}$ and $K_{i}:=I_{i} J_{i}$,

$$
\operatorname{End}_{U_{i}}:=\left\{\alpha I_{i}+\beta J_{i}+\gamma K_{i}: \alpha, \beta, \gamma \in \mathbb{R}\right\}
$$

are the same on all of $M$.

A Riemannian metric $g$ is quaternionic-Hermitian if $g$ is Hermitian for each $I$ and $J$. A manifold $(M, g)$ equipped with an almost quaternionic structure with $g$ quaternionic-Hermitian is called almost-quaternionic-Hermitian.

DEFINITION 2.2. The standard enhanced quaternionic structure of $\mathbb{H}^{m}$ is the 3 -dimensional subspace $Q_{0}$ of the space $\operatorname{End}_{\mathbb{R}} \mathbb{H}^{m}$ spanned by (any) triple $\left(I_{1}, I_{2}, I_{3}\right)$ as above, called an admissible hypercomplex base of $Q_{0}$ (we will also write $\left.\left(I_{1}, I_{2}, I_{3}\right) \in Q_{0}\right)$. 
Definition 2.3. Let $\left(M^{4 m}, g\right)$ and $\left(N^{4 n}, h\right)$ be two almost-quaternionicHermitian manifolds and $\Phi:\left(M^{4 m}, g\right) \rightarrow\left(N^{4 n}, h\right)$ a smooth map. Then $\Phi$ is called $Q$-holomorphic if for every $p \in M^{4 m}$ and each hypercomplex base $\left(I_{1}^{\prime}, I_{2}^{\prime}, I_{3}^{\prime}\right) \in Q_{p}^{M}$ there exists a hypercomplex base $\left(I_{1}, I_{2}, I_{3}\right) \in Q_{\Phi(p)}^{N}$ such that

$$
I_{a}\left(\Phi_{*}\right)_{\mid p}=\left(\Phi_{*}\right)_{\mid p} I_{a}^{\prime} \quad \text { for } a=1,2,3 .
$$

Let $\Phi:\left(M^{4 m}, g\right) \rightarrow\left(N^{4 n}, h\right)$ be a $C^{\infty}$-mapping between two almostquaternionic-Hermitian manifolds of dimensions $4 m$ and $4 n$, respectively. We can regard the quaternionic extension $d^{\mathbb{H}} \Phi$ of the differential $d \Phi$ as a section of the bundle $\left(\Phi^{-1} T^{\mathbb{H}} N\right) \otimes_{\mathbb{H}}\left(T^{\mathbb{H}} M\right)^{*}$ over $M$.

Let $p \in M$. It is well known (see e.g. [3]) that $T_{p}^{\mathbb{H}} M:=T_{p} M \otimes_{\mathbb{R}} \mathbb{H}$ can be decomposed in the following way:

$$
T_{p}^{\mathbb{H}} M:=U_{p}^{\mathbb{H}} \oplus \tau_{1} U_{p}^{\mathbb{H}} \oplus \tau_{2} U_{p}^{\mathbb{H}} \oplus \tau_{3} U_{p}^{\mathbb{H}},
$$

where $\tau_{1}, \tau_{2}, \tau_{3}$ are the semi-involutions defined on $T_{p}^{\mathbb{H}} M$ as

$$
\tau_{1}=\mathrm{id} \otimes \sigma_{1}, \quad \tau_{2}=\mathrm{id} \otimes \sigma_{2}, \quad \tau_{3}=\mathrm{id} \otimes \sigma_{3}
$$

and $\sigma_{1}, \sigma_{2}, \sigma_{3}$ are the involutions of the algebra of quaternions $\mathbb{H}$ defined above.

Take $p \in M$ and let $U$ be a small open neighbourhood of $p$. On $U$, by Definition 2.1, there are almost complex structures $I, J$ and $K:=I J$. Using $I, J$ and $K$ we can define $U_{p}^{\mathbb{H}}$ by

$$
U_{p}^{\mathbb{H}}:=\left\{X+i I X+j J X+k K X: X \in T_{p} M\right\} .
$$

Then we get

$$
\begin{aligned}
& \tau_{1} U_{p}^{\mathbb{H}}=\left\{X+i I X-j J X-k K X: X \in T_{p} M\right\}, \\
& \tau_{2} U_{p}^{\mathbb{H}}=\left\{X-i I X+j J X-k K X: X \in T_{p} M\right\}, \\
& \tau_{3} U_{p}^{\mathbb{H}}=\left\{X-i I X-j J X+k K X: X \in T_{p} M\right\} .
\end{aligned}
$$

R e mark 2.1. There exist elements $X_{1}, \ldots, X_{m}$ of $T_{p} M$ such that the system $\left(X_{1}, \ldots, X_{m}, I X_{1}, \ldots, I X_{m}, J X_{1}, \ldots, J X_{m}, K X_{1}, \ldots, K X_{m}\right)$ forms an orthonormal basis for $T_{p}^{\mathbb{H}} M$ with respect to the metric $g$.

Proof. It is analogous to that in the complex case [8].

Let $\left(x_{0}^{i}, x_{1}^{i}, x_{2}^{i}, x_{3}^{i}\right), i=1, \ldots, m$, be local real coordinates at the point $p$. We can introduce the quaternionic coordinates $\left(q_{1}, \ldots, q_{m}\right)$ as follows:

$$
q_{k}:=x_{0}^{k}+i x_{1}^{k}+j x_{2}^{k}+k x_{3}^{k}, \quad k=1, \ldots, m .
$$

If the almost quaternionic structure $(I, J, K)$ is integrable then we can assume that $I, J$ and $K$ are given by 


$$
\begin{array}{lll}
I\left(\frac{\partial}{\partial x_{0 \mid p}^{k}}\right)=-\frac{\partial}{\partial x_{1 \mid p}^{k}}, & J\left(\frac{\partial}{\partial x_{0 \mid p}^{k}}\right)=-\frac{\partial}{\partial x_{2 \mid p}^{k}}, \quad K\left(\frac{\partial}{\partial x_{0 \mid p}^{k}}\right)=-\frac{\partial}{\partial x_{3 \mid p}^{k}}, \\
I\left(\frac{\partial}{\partial x_{1 \mid p}^{k}}\right)=\frac{\partial}{\partial x_{0 \mid p}^{k}}, \quad J\left(\frac{\partial}{\partial x_{1 \mid p}^{k}}\right)=\frac{\partial}{\partial x_{3 \mid p}^{k}}, \quad K\left(\frac{\partial}{\partial x_{1 \mid p}^{k}}\right)=-\frac{\partial}{\partial x_{2 \mid p}^{k}}, \\
I\left(\frac{\partial}{\partial x_{2 \mid p}^{k}}\right)=-\frac{\partial}{\partial x_{3 \mid p}^{k}}, \quad J\left(\frac{\partial}{\partial x_{2 \mid p}^{k}}\right)=\frac{\partial}{\partial x_{0 \mid p}^{k}}, \quad K\left(\frac{\partial}{\partial x_{2 \mid p}^{k}}\right)=\frac{\partial}{\partial x_{1 \mid p}^{k}}, \\
I\left(\frac{\partial}{\partial x_{3 \mid p}^{k}}\right)=\frac{\partial}{\partial x_{2 \mid p}^{k}}, \quad J\left(\frac{\partial}{\partial x_{3 \mid p}^{k}}\right)=-\frac{\partial}{\partial x_{1 \mid p}^{k}}, \quad K\left(\frac{\partial}{\partial x_{3 \mid p}^{k}}\right)=\frac{\partial}{\partial x_{0 \mid p}^{k}} .
\end{array}
$$

It follows that

$$
\begin{aligned}
& \frac{\partial}{\partial q_{k \mid p}}=\frac{1}{4}\left(\frac{\partial}{\partial x_{0 \mid p}^{k}}-i \frac{\partial}{\partial x_{1 \mid p}^{k}}-j \frac{\partial}{\partial x_{\mid p p}^{k}}-k \frac{\partial}{\partial x_{3 \mid p}^{k}}\right), \\
& \frac{\partial}{\partial q_{k \mid p}^{1}}=\frac{1}{4}\left(\frac{\partial}{\partial x_{0 \mid p}^{k}}-i \frac{\partial}{\partial x_{1 \mid p}^{k}}+j \frac{\partial}{\partial x_{2 \mid p}^{k}}+k \frac{\partial}{\partial x_{3 \mid p}^{k}}\right), \\
& \frac{\partial}{\partial q_{k \mid p}^{2}}=\frac{1}{4}\left(\frac{\partial}{\partial x_{0 \mid p}^{k}}+i \frac{\partial}{\partial x_{\mid p}^{k}}-j \frac{\partial}{\partial x_{\mid p p}^{k}}+k \frac{\partial}{\partial x_{3 \mid p}^{k}}\right), \\
& \frac{\partial}{\partial q_{k \mid p}^{3}}=\frac{1}{4}\left(\frac{\partial}{\partial x_{0 \mid p}^{k}}+i \frac{\partial}{\partial x_{1 \mid p}^{k}}+j \frac{\partial}{\partial x_{2 \mid p}^{k}}-k \frac{\partial}{\partial x_{3 \mid p}^{k}}\right) .
\end{aligned}
$$

Then the system $\left\{\partial / \partial q_{1 \mid p}, \ldots, \partial / \partial q_{m \mid p}\right\}$ forms a basis for $U_{p}^{\mathbb{H}}$ and $\left\{\partial / \partial q_{1 \mid p}^{a}, \ldots, \partial / \partial q_{m \mid p}^{a}\right\}$ are bases for $\tau_{a} U_{p}^{\mathbb{H}}, a=1,2,3$. It is also clear that $\left\{d q_{1 \mid p}, \ldots, d q_{m \mid p}\right\}$ and $\left\{d q_{1 \mid p}^{a}, \ldots, d q_{m \mid p}^{a}\right\}$ are bases for $\left(U_{p}^{\mathbb{H}}\right)^{*}$ and $\left(\tau_{a} U_{p}^{\mathbb{H}}\right)^{*}$, $a=1,2,3$, respectively.

3. Main theorems. Let $V$ be a real vector space of dimension $4 n$. A quaternionic structure in $V$ corresponds to the following decomposition (see Sect. 2):

$$
V^{\mathbb{H}}=U^{\mathbb{H}} \oplus \tau_{1} U^{\mathbb{H}} \oplus \tau_{2} U^{\mathbb{H}} \oplus \tau_{3} U^{\mathbb{H}} .
$$

Consider the tensor products

$$
V^{\mathbb{H}} \otimes_{\mathbb{H}}\left(V^{\mathbb{H}}\right)^{*}, \quad V^{\mathbb{H}} \otimes_{\mathbb{H}} \overline{V^{\mathbb{H}}}, \quad \overline{\left(V^{\mathbb{H}}\right)^{*}} \otimes_{\mathbb{H}}\left(V^{\mathbb{H}}\right)^{*}, \ldots
$$

Each of them can be decomposed into the direct sum of 16 parts with respect to the given quaternionic structure in $V$. In particular, to every real covariant 2-tensor $S$ on $V, S \in V^{*} \otimes_{\mathbb{R}} V^{*}$, there corresponds a 2-tensor $S^{\mathbb{H}} \in \overline{\left(V^{\mathbb{H}}\right)^{*}} \otimes_{\mathbb{H}}$ $\left(V^{\mathbb{H}}\right)^{*}$ which can be decomposed into 4 parts, namely real tensors $S_{0}^{\mathbb{H}}, S_{1}^{\mathbb{H}}$, $S_{2}^{\mathbb{H I}}$ and $S_{3}^{\mathbb{H I}}$ (of genus $0,1,2,3$, respectively) in the following way:

$$
\begin{aligned}
S_{0}^{\mathbb{H}} \in & \overline{\left(U^{\mathbb{H}}\right)^{*}} \otimes_{\mathbb{H}}\left(U^{\mathbb{H}}\right)^{*}+\left(\tau_{1} \overline{U^{\mathbb{H}}}\right)^{*} \otimes_{\mathbb{H}}\left(\tau_{1} U^{\mathbb{H}}\right)^{*}+\left(\tau_{2} \overline{U^{\mathbb{H}}}\right)^{*} \otimes_{\mathbb{H}}\left(\tau_{2} U^{\mathbb{H}}\right)^{*} \\
& +\left(\tau_{3} \overline{U^{\mathbb{H}}}\right)^{*} \otimes_{\mathbb{H}}\left(\tau_{3} U^{\mathbb{H}}\right)^{*},
\end{aligned}
$$




$$
\begin{aligned}
S_{1}^{\mathbb{H}} \in & \overline{\left(U^{\mathbb{H}}\right)^{*}} \otimes_{\mathbb{H}}\left(\tau_{1} U^{\mathbb{H}}\right)^{*}+\left(\tau_{1} \overline{U^{\mathbb{H}}}\right)^{*} \otimes_{\mathbb{H}}\left(U^{\mathbb{H}}\right)^{*}+\left(\tau_{2} \overline{U^{\mathbb{H}}}\right)^{*} \otimes_{\mathbb{H}}\left(\tau_{3} U^{\mathbb{H}}\right)^{*} \\
& +\left(\tau_{3} \overline{U^{\mathbb{H}}}\right)^{*} \otimes_{\mathbb{H}}\left(\tau_{2} U^{\mathbb{H}}\right)^{*}, \\
S_{2}^{\mathbb{H}} \in & \overline{\left(U^{\mathbb{H}}\right)^{*}} \otimes_{\mathbb{H}}\left(\tau_{2} U^{\mathbb{H}}\right)^{*}+\left(\tau_{2} \overline{U^{\mathbb{H}}}\right)^{*} \otimes_{\mathbb{H}}\left(U^{\mathbb{H}}\right)^{*}+\left(\tau_{3} \overline{U^{\mathbb{H}}}\right)^{*} \otimes_{\mathbb{H}}\left(\tau_{1} U^{\mathbb{H}}\right)^{*} \\
& +\left(\tau_{1} \overline{U^{\mathbb{H}}}\right)^{*} \otimes_{\mathbb{H}}\left(\tau_{3} U^{\mathbb{H}}\right)^{*}, \\
S_{3}^{\mathbb{H}} \in & \overline{\left(U^{\mathbb{H}}\right)^{*}} \otimes_{\mathbb{H}}\left(\tau_{3} U^{\mathbb{H}}\right)^{*}+\left(\tau_{3} \overline{U^{\mathbb{H}}}\right)^{*} \otimes_{\mathbb{H}}\left(U^{\mathbb{H}}\right)^{*}+\left(\tau_{1} \overline{U^{\mathbb{H}}}\right)^{*} \otimes_{\mathbb{H}}\left(\tau_{2} U^{\mathbb{H}}\right)^{*} \\
& +\left(\tau_{2} \overline{U^{\mathbb{H}}}\right)^{*} \otimes_{\mathbb{H}}\left(\tau_{1} U^{\mathbb{H}}\right)^{*} .
\end{aligned}
$$

In each line the tensors are mutally biconjugate.

Assume that

$$
\operatorname{dim}_{\mathbb{R}} V=4 .
$$

Let $x \in V$ and $\left(\partial / \partial x^{0}{ }_{\mid x}, \partial / \partial x^{1}{ }_{\mid x}, \partial / \partial x^{2}{ }_{\mid x}, \partial / \partial x^{3}{ }_{\mid x}\right)$ be a base of the tangent space $T_{x} V$ (this base is good for every $x \in V$ and because $T_{x} V \cong V$, we can treat it as a base for $V)$ and let $\left(d x^{0}, d x^{1}, d x^{2}, d x^{3}\right)$ be the dual base. Consider a real symmetric 2-tensor on $V$ :

$$
S=d x^{i} S_{i j} d x^{j} .
$$

The quaternionic decomposition of $S^{\mathbb{H}}$ in pure components looks as follows:

$$
\begin{aligned}
S^{\mathbb{H}}= & d \bar{q} s_{00} d q+d \overline{q^{1}} s_{11} d q^{1}+\overline{d q^{2}} s_{22} d q^{2}+d \overline{q^{3}} s_{33} d q^{3} \\
& +d \bar{q} s_{01} d q^{1}+\overline{d q^{1}} s_{10} d q+\overline{d q^{2}} s_{23} d q^{3}+d \overline{q^{3}} s_{32} d q^{2} \\
& +d \bar{q} s_{02} d q^{2}+d \overline{q^{2}} s_{20} d q+d \overline{q^{1}} s_{13} d q^{3}+d \overline{q^{3}} s_{31} d q^{1} \\
& +d \bar{q} s_{03} d q^{3}+d \overline{q^{3}} s_{30} d q+\overline{d q^{1}} s_{12} d q^{2}+\overline{d q^{2}} s_{21} d q^{1}
\end{aligned}
$$

where

$$
\begin{aligned}
& s_{00}=s_{11}=s_{22}=s_{33} \in \mathbb{R}, \\
& s_{10}=\left(s_{01}\right)^{1}, \quad s_{23}=\tau_{2} s_{01}, \quad s_{32}=\tau_{2} \bar{s}_{01}, \quad s_{23}=\left(s_{32}\right)^{1} \text {, } \\
& s_{20}=\left(s_{02}\right)^{2}, \quad s_{13}=\tau_{1} s_{02}, \quad s_{31}=\tau_{1} \bar{s}_{02}, \quad s_{21}=\left(s_{12}\right)^{3} \text {, } \\
& s_{30}=\left(s_{03}\right)^{3}, \quad s_{12}=\tau_{3} s_{03}, \quad s_{21}=\tau_{3} \bar{s}_{03}, \quad s_{31}=\left(s_{13}\right)^{2} .
\end{aligned}
$$

The relationship between the quaternionic components $s_{i j}$ and the real components $S_{m n}$ is

$$
\begin{aligned}
& \frac{1}{4} S_{00}=s_{00}+\operatorname{Re}\left(s_{01}+s_{02}+s_{03}\right), \\
& \frac{1}{4} S_{11}=s_{00}+\operatorname{Re}\left(s_{01}-s_{02}-s_{03}\right), \\
& \frac{1}{4} S_{22}=s_{00}+\operatorname{Re}\left(-s_{01}+s_{02}-s_{03}\right), \\
& \frac{1}{4} S_{33}=s_{00}+\operatorname{Re}\left(-s_{01}-s_{02}+s_{03}\right), \\
& \frac{1}{4} S_{01}=-\operatorname{Re}\left[\left(s_{02}+s_{03}\right) i\right]=-\operatorname{Re}\left[\left( \pm s_{01}+s_{02}+s_{03}\right) i\right],
\end{aligned}
$$




$$
\begin{aligned}
& \frac{1}{4} S_{02}=-\operatorname{Re}\left[\left(s_{01}+s_{03}\right) j\right]=-\operatorname{Re}\left[\left(s_{01} \pm s_{02}+s_{03}\right) j\right], \\
& \frac{1}{4} S_{03}=-\operatorname{Re}\left[\left(s_{01}+s_{02}\right) k\right]=-\operatorname{Re}\left[\left(s_{01}+s_{02} \pm s_{03}\right) k\right], \\
& \frac{1}{4} S_{12}=-\operatorname{Re}\left[\left(s_{01}-s_{02}\right) k\right]=-\operatorname{Re}\left[\left(s_{01}-s_{02} \pm s_{03}\right) k\right], \\
& \frac{1}{4} S_{13}=-\operatorname{Re}\left[-\left(s_{01}-s_{03}\right) j\right]=-\operatorname{Re}\left[\left(-s_{01} \pm s_{02}+s_{03}\right) j\right], \\
& \frac{1}{4} S_{23}=-\operatorname{Re}\left[-\left(s_{02}-s_{03}\right) i\right]=-\operatorname{Re}\left[\left( \pm s_{01}-s_{02}+s_{03}\right) i\right] .
\end{aligned}
$$

Writing $\pm s_{0 k}$ we indicate that we are free to choose the sign due to the fact that $\operatorname{Re}\left(s_{01} i\right)=\operatorname{Re}\left(s_{02} j\right)=\operatorname{Re}\left(s_{03} k\right)=0$.

On the other hand, we have

$$
\begin{aligned}
& s_{00}=\frac{1}{16}\left(S_{00}+S_{11}+S_{22}+S_{33}\right), \\
& s_{01}=\frac{1}{16}\left(S_{00}+S_{11}-S_{22}-S_{33}\right)+\frac{1}{8}\left(S_{02}-S_{13}\right) j+\frac{1}{8}\left(S_{03}+S_{12}\right) k, \\
& s_{02}=\frac{1}{16}\left(S_{00}-S_{11}+S_{22}-S_{33}\right)+\frac{1}{8}\left(S_{01}+S_{23}\right) i+\frac{1}{8}\left(S_{03}-S_{12}\right) k, \\
& s_{03}=\frac{1}{16}\left(S_{00}-S_{11}-S_{22}+S_{33}\right)+\frac{1}{8}\left(S_{01}-S_{23}\right) i+\frac{1}{8}\left(S_{02}+S_{13}\right) j .
\end{aligned}
$$

Hereafter we will consider a real Riemannian manifold $M$ which is locally conformally flat with $\operatorname{dim}_{\mathbb{R}} M=4$ (e.g. the sphere $S^{4} \cong \mathbb{H} \mathbb{P}^{1}$ or the torus $T^{4} \cong \mathbb{H} / \mathbb{Z}^{4}$, see e.g. [2]). Then we can assume that in a neighbourhood of every point $p$ of $M$ there exists a system of local coordinates $\left(x^{0}, x^{1}, x^{2}, x^{3}\right)$ such that the metric $g$ is expressed by

$$
g=g_{\mathbb{R}}^{0}\left[\left(d x^{0}\right)^{2}+\left(d x^{1}\right)^{2}+\left(d x^{2}\right)^{2}+\left(d x^{3}\right)^{2}\right],
$$

where $g_{\mathbb{R}}^{0}$ is a real positive $C^{\infty}$-function defined about $p$. Consider the quaternionic coordinate $q:=x^{0}+i x^{1}+j x^{2}+k x^{3}$ associated with the given system of real coordinates. Then the quaternionic expression of $g$ is

$$
g=g_{\mathbb{H}}^{0}\left[d \bar{q} \otimes d q+d \overline{q^{1}} \otimes d q^{1}+d \overline{q^{2}} \otimes d q^{2}+d \overline{q^{3}} \otimes d q^{3}\right] .
$$

Comparing the expressions for $g$ in real and quaternionic coordinates we get:

Proposition 3.1. $g_{\mathbb{R}}^{0}=4 g_{\mathbb{H}}^{0}$.

Pr o of. This follows by straightforward calculations.

Definition 3.1 [5]. Let $\Phi:(M, g) \rightarrow(N, h)$ be a smooth map between Riemannian manifolds. The stress-energy tensor of $\Phi$ is the symmetric 2tensor on $M$ given by

$$
S(\Phi):=e \cdot g-\Phi^{*} h,
$$

where $e=e(\Phi)$ denotes the energy density of $\Phi$ :

$$
e(\Phi):=\frac{1}{2}|d \Phi|^{2}=\frac{1}{2} g^{i j} h_{\alpha \beta} \Phi_{i}^{\alpha} \Phi_{j}^{\beta},
$$

and $\left(\Phi_{i}^{\alpha}\right)=\left(\partial \Phi^{\alpha} / \partial x^{i}\right)$ is a local representation of $d \Phi$.

We will compute the quaternionic components of $S(\Phi)$. 
I. Computation of $\Phi^{*} h$. The real expression of the metric $h$ on $N$ is

$$
h=\sum_{\alpha, \beta} h_{\alpha \beta} d y^{\alpha} \otimes d y^{\beta} \quad\left(h_{\alpha \beta}=h_{\beta \alpha}\right),
$$

where $\left(y^{\alpha}\right)$ is a local system of real coordinates defined in an open neighbourhood of $\Phi(p)$, with $p$ a fixed point in $M$. If $\left(x^{j}\right)$ is a system of local coordinates about $p$ then

$$
\Phi^{*} h=d x^{i} \Phi_{i}^{\alpha} h_{\alpha \beta} \Phi_{j}^{\beta} d x^{j} .
$$

In order to pass to quaternionic coordinates we have to consider the extension of the metric $h$ to the quaternionified tangent bundle of $N, T^{\mathbb{H}} N:=$ $T N \otimes_{\mathbb{R}} \mathbb{H}$. If $X^{\mathbb{H}}, Y^{\mathbb{H}} \in T_{p}^{\mathbb{H}} N$ then

$$
X^{\mathbb{H}}=\left(X^{1}, \ldots, X^{n}\right), \quad Y^{\mathbb{H}}=\left(Y^{1}, \ldots, Y^{n}\right),
$$

where $4 n=\operatorname{dim}_{\mathbb{R}} N$ and

$$
X^{\alpha}=X_{0}^{\alpha}+i X_{1}^{\alpha}+j X_{2}^{\alpha}+k X_{3}^{\alpha}, \quad Y^{\beta}=Y_{0}^{\beta}+i Y_{1}^{\beta}+j Y_{2}^{\beta}+k Y_{3}^{\beta} .
$$

Then we set

$$
\begin{aligned}
\left\langle X^{\mathbb{H}}, Y^{\mathbb{H}}\right\rangle & =\left\langle X^{\mathbb{H}}, Y^{\mathbb{H}}\right\rangle_{h} \\
& :=\sum_{\alpha, \beta=1}^{n}\left(X_{0}^{\alpha}+i X_{1}^{\alpha}+j X_{2}^{\alpha}+k X_{3}^{\alpha}\right) h_{\alpha \beta}\left(Y_{0}^{\beta}+i Y_{1}^{\beta}+j Y_{2}^{\beta}+k Y_{3}^{\beta}\right) .
\end{aligned}
$$

Taking into account (2.1) we can write

$$
\begin{aligned}
\Phi^{*} h= & \left(d \bar{q} \frac{\partial \Phi^{\alpha}}{\partial \bar{q}}+d \overline{q^{1}} \frac{\partial \Phi^{\alpha}}{\partial \overline{q^{1}}}+d \overline{q^{2}} \frac{\partial \Phi^{\alpha}}{\partial \overline{q^{2}}}+d \overline{q^{3}} \frac{\partial \Phi^{\alpha}}{\partial \overline{q^{3}}}\right) h_{\alpha \beta} \\
& \times\left(\frac{\partial \Phi^{\beta}}{\partial q} d q+\frac{\partial \Phi^{\beta}}{\partial q^{1}} d q^{1}+\frac{\partial \Phi^{\beta}}{\partial q^{2}} d q^{2}+\frac{\partial \Phi^{\beta}}{\partial q^{3}} d q^{3}\right) .
\end{aligned}
$$

Finally, we get

$$
\begin{aligned}
\Phi^{*} h= & \left\langle\Phi_{\bar{q}}, \Phi_{q}\right\rangle\left(d \bar{q} d q+\overline{d q^{1}} d q^{1}+d \overline{q^{2}} d q^{2}+d \overline{q^{3}} d q^{3}\right) \\
& +\left[d \bar{q}\left\langle\Phi_{\bar{q}}, \Phi_{q^{1}}\right\rangle d q^{1}+d \overline{q^{1}}\left\langle\Phi_{\bar{q}}, \Phi_{q^{1}}\right\rangle^{1} d q+d \overline{q^{2}}\left\langle\Phi_{\bar{q}}, \Phi_{q^{1}}\right\rangle^{2} d q^{3}\right. \\
& \left.+d \overline{q^{3}}\left\langle\Phi_{\bar{q}}, \Phi_{q^{1}}\right\rangle^{3} d q^{2}\right] \\
& +\left[d \bar{q}\left\langle\Phi_{\bar{q}}, \Phi_{q^{2}}\right\rangle d q^{2}+d \overline{q^{2}}\left\langle\Phi_{\bar{q}}, \Phi_{q^{2}}\right\rangle^{2} d q+d \overline{q^{1}}\left\langle\Phi_{\bar{q}}, \Phi_{q^{2}}\right\rangle^{1} d q^{3}\right. \\
& \left.+d \overline{q^{3}}\left\langle\Phi_{\bar{q}}, \Phi_{q^{2}}\right\rangle^{3} d q^{1}\right] \\
& +\left[d \bar{q}\left\langle\Phi_{\bar{q}}, \Phi_{q^{3}}\right\rangle d q^{3}+d \overline{q^{3}}\left\langle\Phi_{\bar{q}}, \Phi_{q^{3}}\right\rangle^{3} d q+d \overline{q^{1}}\left\langle\Phi_{\bar{q}}, \Phi_{q^{3}}\right\rangle^{1} d q^{2}\right. \\
& \left.+d \overline{q^{2}}\left\langle\Phi_{\bar{q}}, \Phi_{q^{3}}\right\rangle^{2} d q^{1}\right] .
\end{aligned}
$$


II. Computation of $e(\Phi) \cdot g$. By the definition we have

$$
e(\Phi)=\frac{1}{2}|d \Phi|^{2}=\frac{1}{2} g^{i j} h_{\alpha \beta} \Phi_{i}^{\alpha} \Phi_{j}^{\beta}=\frac{1}{2} g_{\mathbb{H}}^{0}\left(\left|\Phi_{0}\right|^{2}+\left|\Phi_{1}\right|^{2}+\left|\Phi_{2}\right|^{2}+\left|\Phi_{3}\right|^{2}\right) .
$$

Then

$$
\begin{aligned}
e(\Phi) \cdot g & =\frac{1}{8}\left(\left|\Phi_{0}\right|^{2}+\left|\Phi_{1}\right|^{2}+\left|\Phi_{2}\right|^{2}+\left|\Phi_{3}\right|^{2}\right)\left(d \bar{q} d q+\overline{d q^{1}} d q^{1}+\overline{d q^{2}} d q^{2}+d \overline{q^{3}} d q^{3}\right) \\
& =2\left\langle\Phi_{\bar{q}}, \Phi_{q}\right\rangle\left(d \bar{q} d q+\overline{q^{1}} d q^{1}+d \overline{q^{2}} d q^{2}+d \overline{q^{3}} d q^{3}\right) .
\end{aligned}
$$

Now, we can write an explicit expression for the stress-energy tensor of $\Phi$ :

$$
\begin{aligned}
S(\Phi)= & \left\langle\Phi_{\bar{q}}, \Phi_{q}\right\rangle\left(d \bar{q} d q+d \overline{q^{1}} d q^{1}+d \overline{q^{2}} d q^{2}+d \overline{q^{3}} d q^{3}\right) \\
& -\left[d \bar{q}\left\langle\Phi_{\bar{q}}, \Phi_{q^{1}}\right\rangle d q^{1}+d \overline{q^{1}}\left\langle\Phi_{\bar{q}}, \Phi_{q^{1}}\right\rangle^{1} d q+d \overline{q^{2}}\left\langle\Phi_{\bar{q}}, \Phi_{q^{1}}\right\rangle^{2} d q^{3}\right. \\
& \left.+d \overline{q^{3}}\left\langle\Phi_{\bar{q}}, \Phi_{q^{1}}\right\rangle^{3} d q^{2}\right] \\
& -\left[d \bar{q}\left\langle\Phi_{\bar{q}}, \Phi_{q^{2}}\right\rangle d q^{2}+d \overline{q^{2}}\left\langle\Phi_{\bar{q}}, \Phi_{q^{2}}\right\rangle^{2} d q+d \overline{q^{1}}\left\langle\Phi_{\bar{q}}, \Phi_{q^{2}}\right\rangle^{1} d q^{3}\right. \\
& \left.+d \overline{q^{3}}\left\langle\Phi_{\bar{q}}, \Phi_{q^{2}}\right\rangle^{3} d q^{1}\right] \\
& -\left[d \bar{q}\left\langle\Phi_{\bar{q}}, \Phi_{q^{3}}\right\rangle d q^{3}+d \overline{q^{3}}\left\langle\Phi_{\bar{q}}, \Phi_{q^{3}}\right\rangle^{3} d q+d \overline{q^{1}}\left\langle\Phi_{\bar{q}}, \Phi_{q^{3}}\right\rangle^{1} d q^{2}\right. \\
& \left.+d \overline{q^{2}}\left\langle\Phi_{\bar{q}}, \Phi_{q^{3}}\right\rangle^{2} d q^{1}\right] .
\end{aligned}
$$

According to the above decomposition we can define four tensors $S_{0}, S_{1}$, $S_{2}, S_{3}$ so that every square bracket [.] corresponds to one component of the decomposition of $S(\Phi)$ in these tensors: $S(\Phi)=S_{0}+S_{1}+S_{2}+S_{3}$.

Proposition 3.2. ( $\Phi$ is conformal $) \Leftrightarrow\left(S_{1}=S_{2}=S_{3}=0\right)$.

Proof. Notice that the equations

$$
\left\langle\Phi_{\bar{q}}, \Phi_{q^{1}}\right\rangle=\left\langle\Phi_{\bar{q}}, \Phi_{q^{2}}\right\rangle=\left\langle\Phi_{\bar{q}}, \Phi_{q^{3}}\right\rangle=0,
$$

which express the vanishing of the components in $S_{1}, S_{2}$ and $S_{3}$, are equivalent to the conditions

$$
\begin{gathered}
\left|\Phi_{0}\right|^{2}=\left|\Phi_{1}\right|^{2}=\left|\Phi_{2}\right|^{2}=\left|\Phi_{3}\right|^{2}, \\
\left\langle\Phi_{0}, \Phi_{i}\right\rangle=0, \quad i=1,2,3 ; \quad\left\langle\Phi_{i}, \Phi_{j}\right\rangle=0, \quad i \neq j, i, j \neq 0,
\end{gathered}
$$

which just express the conformality of $\Phi$.

Remark 3.1. ( $\Phi$ is conformal) $\Leftrightarrow(S(\Phi)$ is pure of genus 0$)$.

Corollary 3.1. If $\Phi$ is locally regular, i.e. $\Phi_{\bar{q}}=0$, then $S(\Phi)=0$.

Corollary 3.2. $S(\Phi)=0$ if and only if $\Phi=$ const.

Proof. By (3.2), $S(\Phi)=0$ if and only if

$$
\left\langle\Phi_{\bar{q}}, \Phi_{q}\right\rangle=\left\langle\Phi_{\bar{q}}, \Phi_{q^{1}}\right\rangle=\left\langle\Phi_{\bar{q}}, \Phi_{q^{2}}\right\rangle=\left\langle\Phi_{\bar{q}}, \Phi_{q^{3}}\right\rangle=0 .
$$


By straightforward calculations the above equalities are equivalent to

$$
\left|\Phi_{0}\right|^{2}=\left|\Phi_{1}\right|^{2}=\left|\Phi_{2}\right|^{2}=\left|\Phi_{3}\right|^{2}=0 \quad \text { and } \quad\left\langle\Phi_{i}, \Phi_{k}\right\rangle=0, \quad i, k=0,1,2,3 .
$$

But this is possible if and only if $\Phi$ is a constant.

DeFinition 3.2. A 2-tensor $S$ defined on an almost quaternionic manifold $\left(M^{4}, g\right)$ with $\operatorname{dim}_{\mathbb{R}} M^{4}=4$ and standard enhanced quaternionic structure $Q_{0}$ is Hermitian if for any $p \in M^{4}$ we have

$$
S\left(I_{\alpha} X, I_{\alpha} Y\right)=S(X, Y) \quad \text { for } \alpha=1,2,3,
$$

where $\left(I_{1}, I_{2}, I_{3}\right) \in Q_{0 \mid p}$ and $X, Y \in T_{p} M^{4}$.

Remark 3.2. ( $S$ is pure of genus 0$) \Leftrightarrow(S$ is Hermitian).

Remark 3.3. ( $\Phi$ is conformal) $\Leftrightarrow(S(\Phi)$ is Hermitian).

Proposition 3.3. Let $\Phi:\left(M^{4}, g\right) \rightarrow\left(N^{4 n}, h\right)$ be a smooth mapping between two almost-quaternionic-Hermitian manifolds. Assume that $\left(M^{4}, g\right)$ is locally conformally flat. If $\Phi$ is Q-holomorphic then $S(\Phi)$ is Hermitian.

Pro of. By the definition of $S(\Phi)$ it is enough to show that $\Phi^{*} h$ is Hermitian on $\left(M^{4}, g\right)$. Indeed,

$$
\begin{aligned}
\Phi^{*} h\left(I_{\alpha} X, I_{\alpha} Y\right) & =h\left(d \Phi\left(I_{\alpha} X\right), d \Phi\left(I_{\alpha} Y\right)\right)=h\left(I_{\alpha}^{\prime}\left(d \Phi(X), I_{\alpha}^{\prime}(d \Phi(Y))\right)\right. \\
& =h(d \Phi(X), d \Phi(Y))=\Phi^{*} h(X, Y) .
\end{aligned}
$$

Proposition 3.4. Let $\Phi:\left(M^{4}, g\right) \rightarrow\left(N^{4 n}, h\right)$ be a smooth mapping between almost quaternionic Hermitian manifolds. Assume that $\left(M^{4}, g\right)$ is locally conformally flat. If $\Phi$ is Q-holomorphic then $\Phi$ is harmonic if and only if it is homothetic.

Proof. Note that

$$
e(\Phi)=2 g_{\mathbb{H}}^{0}\left\langle\Phi_{\bar{q}}, \Phi_{q}\right\rangle .
$$

If $\Phi$ is $Q$-holomorphic then $\Phi$ is conformal. By Proposition 3.2 and (3.2) we get

$$
S(\Phi)=g_{\mathbb{H}}^{0}\left\langle\Phi_{\bar{q}}, \Phi_{q}\right\rangle g
$$

On the other hand, conformality of $\Phi$ means that $\Phi^{*} h=\mu g$ for some continuous and non-negative function $\mu$ defined on $M$. By (3.3) and (3.4) we obtain $\mu=g_{\mathbb{H}}^{0}\left\langle\Phi_{\bar{q}}, \Phi_{q}\right\rangle$. Thus

$$
\begin{aligned}
(\operatorname{div} S(\Phi)=0) & \Leftrightarrow\left[\left\langle d\left(g_{\mathbb{H}}^{0}\left\langle\Phi_{\bar{q}}, \Phi_{q}\right\rangle\right), g\right\rangle=0\right] \Leftrightarrow\left[d\left(g_{\mathbb{H}}^{0}\left\langle\Phi_{\bar{q}}, \Phi_{q}\right\rangle\right)=0\right] \\
& \Leftrightarrow(d \mu=0) \Leftrightarrow(\mu=\mathrm{const}) \Leftrightarrow(\Phi \text { is homothetic }) .
\end{aligned}
$$


If $\Phi$ is homothetic then $\Phi^{*} h=\mu_{0} g$, where $\mu_{0}=$ const. So, we have $S=\left(e-\mu_{0}\right) g$ and $\operatorname{div} S=\langle d e, g\rangle$. But on the other hand, $e=0$, and so $\Phi$ is harmonic.

Recall that if $S$ is a real 2-tensor on a (real) Riemannian manifold $(M, g)$, then one defines the divergence of $S$ (see e.g. [5]) in the local coordinates $\left(x^{i}\right)$ by

$$
\left(\operatorname{div}_{\mathbb{R}} S\right)_{i}=(\operatorname{div} S)_{i}:=g^{j k} \nabla_{\partial_{j}} S_{k i}
$$

Definition 3.3. We define a quaternionic divergence of the quaternionic 2 -tensor $s_{\mathbb{H}}$ by

$$
\left(\operatorname{div}_{\mathbb{H}} s_{\mathbb{H}}\right)_{\gamma}:=g_{\mathbb{H}}^{\alpha \beta} \nabla_{\partial_{\alpha}} s_{\beta \gamma},
$$

where $\alpha, \beta, \gamma$ stand for $q, q^{1}, q^{2}, q^{3}$.

Proposition 3.5. If the metric $g$ is locally conformally flat then

$$
\begin{aligned}
64 \operatorname{Re}\left[\left(\operatorname{div}_{\mathbb{H}} s_{\mathbb{H}}\right)_{q}\right] & =\left(\operatorname{div}_{\mathbb{R}} S\right)_{0}, \\
64 \operatorname{Re}\left[i\left(\operatorname{div}_{\mathbb{H}} s_{\mathbb{H}}\right)_{\overline{1}}\right] & =\left(\operatorname{div}_{\mathbb{R}} S\right)_{1}, \\
64 \operatorname{Re}\left[j\left(\operatorname{div}_{\mathbb{H}} s_{\mathbb{H}}\right)_{\overline{2}}\right] & =\left(\operatorname{div}_{\mathbb{R}} S\right)_{2}, \\
64 \operatorname{Re}\left[k\left(\operatorname{div}_{\mathbb{H}} s_{\mathbb{H}}\right)_{\overline{3}}\right] & =\left(\operatorname{div}_{\mathbb{R}} S\right)_{3},
\end{aligned}
$$

Pr o of. This follows by straightforward computations.

Let us quote a very important result of Eells [5]:

TheOREM 3.1. Suppose that $\Phi:(M, g) \rightarrow(N, h)$ is a smooth mapping between smooth Riemannian manifolds. If $\Phi$ is harmonic then $S(\Phi)$ is conservative (i.e. $\operatorname{div}_{\mathbb{R}} S(\Phi)=0$ ). If $\Phi$ is a differentiable submersion almost everywhere and $\operatorname{div}_{\mathbb{R}} S(\Phi)=0$, then $\Phi$ is harmonic.

Now, we can state

Theorem 3.2. Let $\Phi:\left(M^{4}, g\right) \rightarrow\left(N^{4 n}, h\right)$ be a smooth map between smooth Riemannian manifolds. Assume that $M$ is locally conformally flat. If $\Phi$ is harmonic then $\Phi$ satisfies the following system of real equations:

$$
\begin{aligned}
& \frac{1}{32} \nabla_{\partial_{0}}\left(\left|\Phi_{0}\right|^{2}-\left|\Phi_{1}\right|^{2}-\left|\Phi_{2}\right|^{2}-\left|\Phi_{3}\right|^{2}\right) \\
& +\nabla_{\partial_{1}}\left\langle\Phi_{0}, \Phi_{1}\right\rangle+\nabla_{\partial_{2}}\left\langle\Phi_{0}, \Phi_{2}\right\rangle+\nabla_{\partial_{3}}\left\langle\Phi_{0}, \Phi_{3}\right\rangle=0, \\
& \frac{1}{32} \nabla_{\partial_{1}}\left(\left|\Phi_{0}\right|^{2}-\left|\Phi_{1}\right|^{2}-\left|\Phi_{2}\right|^{2}-\left|\Phi_{3}\right|^{2}\right) \\
& -\nabla_{\partial_{0}}\left\langle\Phi_{0}, \Phi_{1}\right\rangle+\nabla_{\partial_{2}}\left\langle\Phi_{0}, \Phi_{2}\right\rangle-\nabla_{\partial_{3}}\left\langle\Phi_{0}, \Phi_{3}\right\rangle=0, \\
& \frac{1}{32} \nabla_{\partial_{2}}\left(\left|\Phi_{0}\right|^{2}-\left|\Phi_{1}\right|^{2}-\left|\Phi_{2}\right|^{2}-\left|\Phi_{3}\right|^{2}\right) \\
& -\nabla_{\partial_{0}}\left\langle\Phi_{0}, \Phi_{1}\right\rangle-\nabla_{\partial_{1}}\left\langle\Phi_{0}, \Phi_{2}\right\rangle+\nabla_{\partial_{3}}\left\langle\Phi_{0}, \Phi_{3}\right\rangle=0, \\
& \frac{1}{32} \nabla_{\partial_{3}}\left(\left|\Phi_{0}\right|^{2}-\left|\Phi_{1}\right|^{2}-\left|\Phi_{2}\right|^{2}-\left|\Phi_{3}\right|^{2}\right) \\
& -\nabla_{\partial_{0}}\left\langle\Phi_{0}, \Phi_{1}\right\rangle+\nabla_{\partial_{1}}\left\langle\Phi_{0}, \Phi_{2}\right\rangle-\nabla_{\partial_{2}}\left\langle\Phi_{0}, \Phi_{3}\right\rangle=0,
\end{aligned}
$$


where $\Phi_{i}:=\partial \Phi / \partial x^{i}$ and $\langle\cdot, \cdot\rangle$ means a real scalar product. Moreover, if $\Phi$ is a differentiable submersion almost everywhere and the system (3.6) is satisfied then $\Phi$ is harmonic.

P r o of. By the result of Eells, Theorem 3.1, and by Proposition 3.5 the condition $\operatorname{div}_{\mathbb{R}} S(\Phi)=0$ is equivalent to the following system of quaternionic equations:

$$
\begin{array}{rlrl}
\operatorname{Re}\left[\left(\operatorname{div}_{\mathbb{H}} s_{\mathbb{H}}(\Phi)\right)_{0}\right] & =0, \quad & \operatorname{Re}\left[i\left(\operatorname{div}_{\mathbb{H}} s_{\mathbb{H}}(\Phi)\right)_{\overline{1}}\right]=0, \\
\operatorname{Re}\left[j\left(\operatorname{div}_{\mathbb{H}} s_{\mathbb{H}}(\Phi)\right)_{\overline{2}}\right]=0, \quad \operatorname{Re}\left[k\left(\operatorname{div}_{\mathbb{H}} s_{\mathbb{H}}(\Phi)\right)_{\overline{3}}\right]=0,
\end{array}
$$

where $s_{\mathbb{H}}(\Phi)$ denotes the quaternionic stress-energy tensor of $\Phi$.

By the assumption, the metric $g$ has the form (3.1). Hence, the only non-zero quaternionic components of $g$ are $g^{00}, g^{\overline{11}}, g^{\overline{22}}, g^{\overline{33}}$ and they equal $g_{\mathbb{H}}^{0} \neq 0$, which is real. Then we have

$$
\begin{aligned}
\left(\operatorname{div}_{\mathbb{H}} s_{\mathbb{H}}\right)_{0} & =\sum_{\alpha} g^{\alpha \alpha} \nabla_{\partial_{\alpha}} s_{\alpha 0}, \\
\left(\operatorname{div}_{\mathbb{H}} s_{\mathbb{H}}\right)_{\bar{\gamma}} & =\sum_{\alpha} g^{\alpha \alpha} \nabla_{\partial_{\alpha}} s_{\alpha \bar{\gamma}}, \quad \gamma=1,2,3 .
\end{aligned}
$$

Substituting the quaternionic expression $s_{\mathbb{H}}$ for the stress-energy tensor into (3.8) we get

$$
\begin{aligned}
& \frac{\left(\operatorname{div}_{\mathbb{H}} s_{\mathbb{H}}\right)_{0}}{g_{\mathbb{H}}^{0}}=\nabla_{\partial / \partial q}\left\langle\Phi_{\bar{q}}, \Phi_{q}\right\rangle-\nabla_{\partial / \partial q^{1}}\left\langle\Phi_{\bar{q}}, \Phi_{q^{1}}\right\rangle^{1} \\
& -\nabla_{\partial / \partial q^{2}}\left\langle\Phi_{\bar{q}}, \Phi_{q^{2}}\right\rangle^{2}-\nabla_{\partial / \partial q^{3}}\left\langle\Phi_{\bar{q}}, \Phi_{q^{3}}\right\rangle^{3}, \\
& \frac{\left(\operatorname{div}_{\mathbb{H}} s_{\mathbb{H}}\right)_{\overline{1}}}{g_{\mathbb{H}}^{0}}=-\nabla_{\partial / \partial q}\left\langle\Phi_{\bar{q}}, \Phi_{q^{1}}\right\rangle+\nabla_{\partial / \partial q^{1}}\left\langle\Phi_{\bar{q}}, \Phi_{q}\right\rangle \\
& -\nabla_{\partial / \partial q^{2}}\left\langle\Phi_{\bar{q}}, \Phi_{q^{3}}\right\rangle^{2}-\nabla_{\partial / \partial q^{3}}\left\langle\Phi_{\bar{q}}, \Phi_{q^{2}}\right\rangle^{3}, \\
& \frac{\left(\operatorname{div}_{\mathbb{H}} s_{\mathbb{H}}\right)_{\overline{2}}}{g_{\mathbb{H}}^{0}}=-\nabla_{\partial / \partial q}\left\langle\Phi_{\bar{q}}, \Phi_{q^{2}}\right\rangle-\nabla_{\partial / \partial q^{1}}\left\langle\Phi_{\bar{q}}, \Phi_{q^{3}}\right\rangle^{1} \\
& +\nabla_{\partial / \partial q^{2}}\left\langle\Phi_{\bar{q}}, \Phi_{q}\right\rangle-\nabla_{\partial / \partial q^{3}}\left\langle\Phi_{\bar{q}}, \Phi_{q^{1}}\right\rangle^{3}, \\
& \frac{\left(\operatorname{div}_{\mathbb{H}} s_{\mathbb{H}}\right)_{\overline{3}}}{g_{\mathbb{H}}^{0}}=-\nabla_{\partial / \partial q}\left\langle\Phi_{\bar{q}}, \Phi_{q^{3}}\right\rangle-\nabla_{\partial / \partial q^{1}}\left\langle\Phi_{\bar{q}}, \Phi_{q^{2}}\right\rangle^{1} \\
& -\nabla_{\partial / \partial q^{2}}\left\langle\Phi_{\bar{q}}, \Phi_{q^{1}}\right\rangle^{2}+\nabla_{\partial / \partial q^{3}}\left\langle\Phi_{\bar{q}}, \Phi_{q}\right\rangle .
\end{aligned}
$$

Since

for $i, m=0,1,2,3$ and

$$
\nabla_{\partial / \partial q^{i}}\left\langle\Phi_{\bar{q}}, \Phi_{q^{m}}\right\rangle^{i}=\left[\nabla_{\partial / \partial q}\left\langle\Phi_{\bar{q}}, \Phi_{q^{m}}\right\rangle\right]^{i}
$$

$$
\operatorname{Re}\left[\nabla_{\partial / \partial q}\left\langle\Phi_{\bar{q}}, \Phi_{q^{n}}\right\rangle\right]^{n}=\operatorname{Re} \nabla_{\partial / \partial q}\left\langle\Phi_{\bar{q}}, \Phi_{q^{n}}\right\rangle, \quad n=0,1,2,3,
$$

we see that the system (3.7) is equivalent to 


$$
\begin{aligned}
\operatorname{Re}\left[\nabla_{\partial / \partial q}\left\langle\Phi_{\bar{q}}, \Phi_{q}\right\rangle-\nabla_{\partial / \partial q}\left\langle\Phi_{\bar{q}}, \Phi_{q^{1}}\right\rangle\right. & \left.-\nabla_{\partial / \partial q}\left\langle\Phi_{\bar{q}}, \Phi_{q^{2}}\right\rangle-\nabla_{\partial / \partial q}\left\langle\Phi_{\bar{q}}, \Phi_{q^{3}}\right\rangle\right]=0, \\
\operatorname{Re} i\left[\nabla_{\partial / \partial q}\left\langle\Phi_{\bar{q}}, \Phi_{q^{1}}\right\rangle-\right. & \nabla_{\partial / \partial q^{1}}\left\langle\Phi_{\bar{q}}, \Phi_{q}\right\rangle \\
& \left.+\nabla_{\partial / \partial q}\left\langle\Phi_{\bar{q}}, \Phi_{q^{3}}\right\rangle+\nabla_{\partial / \partial q}\left\langle\Phi_{\bar{q}}, \Phi_{q^{2}}\right\rangle\right]=0, \\
\operatorname{Re} j\left[\nabla_{\partial / \partial q}\left\langle\Phi_{\bar{q}}, \Phi_{q^{2}}\right\rangle+\right. & \nabla_{\partial / \partial q}\left\langle\Phi_{\bar{q}}, \Phi_{q^{3}}\right\rangle \\
& \left.-\nabla_{\partial / \partial q^{2}}\left[\Phi_{\bar{q}}, \Phi_{q}\right\rangle+\nabla_{\partial / \partial q}\left\langle\Phi_{\bar{q}}, \Phi_{q^{1}}\right\rangle\right]=0, \\
\operatorname{Re} k\left[\nabla_{\partial / \partial q}\left\langle\Phi_{\bar{q}}, \Phi_{q^{3}}\right\rangle+\right. & \nabla_{\partial / \partial q}\left\langle\Phi_{\bar{q}}, \Phi_{q^{2}}\right\rangle \\
& \left.+\nabla_{\partial / \partial q}\left\langle\Phi_{\bar{q}}, \Phi_{q^{1}}\right\rangle-\nabla_{\partial / \partial q^{3}}\left\langle\Phi_{\bar{q}}, \Phi_{q}\right\rangle\right]=0 .
\end{aligned}
$$

Now, note that

$$
\begin{aligned}
& \operatorname{Re} \nabla_{\partial / \partial q}\left\langle\Phi_{\bar{q}}, \Phi_{q}\right\rangle=\frac{1}{64} \nabla_{\partial_{0}}\left(\left|\Phi_{0}\right|^{2}+\left|\Phi_{1}\right|^{2}+\left|\Phi_{2}\right|^{2}+\left|\Phi_{3}\right|^{2}\right) \\
& \operatorname{Re} \nabla_{\partial / \partial q}\left\langle\Phi_{\bar{q}}, \Phi_{q^{1}}\right\rangle \\
& =\frac{1}{64} \nabla_{\partial_{0}}\left(\left|\Phi_{0}\right|^{2}+\left|\Phi_{1}\right|^{2}-\left|\Phi_{2}\right|^{2}-\left|\Phi_{3}\right|^{2}\right) \\
& \quad+2 \nabla_{\partial_{2}}\left[\left\langle\Phi_{0}, \Phi_{2}\right\rangle-\left\langle\Phi_{1}, \Phi_{3}\right\rangle\right]+2 \nabla_{\partial_{3}}\left[\left\langle\Phi_{0}, \Phi_{3}\right\rangle+\left\langle\Phi_{1}, \Phi_{2}\right\rangle\right], \\
& \operatorname{Re} \nabla_{\partial / \partial q}\left\langle\Phi_{\bar{q}}, \Phi_{q^{2}}\right\rangle \\
& =\frac{1}{64} \nabla_{\partial_{0}}\left(\left|\Phi_{0}\right|^{2}-\left|\Phi_{1}\right|^{2}+\left|\Phi_{2}\right|^{2}-\left|\Phi_{3}\right|^{2}\right) \\
& \quad+2 \nabla_{\partial_{1}}\left[\left\langle\Phi_{0}, \Phi_{1}\right\rangle+\left\langle\Phi_{2}, \Phi_{3}\right\rangle\right]+2 \nabla_{\partial_{3}}\left[\left\langle\Phi_{0}, \Phi_{3}\right\rangle-\left\langle\Phi_{1}, \Phi_{2}\right\rangle\right],
\end{aligned}
$$

$\operatorname{Re} \nabla_{\partial / \partial q}\left\langle\Phi_{\bar{q}}, \Phi_{q^{3}}\right\rangle$

$$
\begin{aligned}
= & \frac{1}{64} \nabla_{\partial_{0}}\left(\left|\Phi_{0}\right|^{2}-\left|\Phi_{1}\right|^{2}-\left|\Phi_{2}\right|^{2}+\left|\Phi_{3}\right|^{2}\right) \\
& +2 \nabla_{\partial_{1}}\left[\left\langle\Phi_{0}, \Phi_{1}\right\rangle-\left\langle\Phi_{2}, \Phi_{3}\right\rangle\right]+2 \nabla_{\partial_{2}}\left[\left\langle\Phi_{0}, \Phi_{2}\right\rangle+\left\langle\Phi_{1}, \Phi_{3}\right\rangle\right],
\end{aligned}
$$

$\operatorname{Re} i \nabla_{\partial / \partial q}\left\langle\Phi_{\bar{q}}, \Phi_{q^{1}}\right\rangle$

$$
\begin{aligned}
= & \frac{1}{64} \nabla_{\partial_{1}}\left(\left|\Phi_{0}\right|^{2}+\left|\Phi_{1}\right|^{2}-\left|\Phi_{2}\right|^{2}-\left|\Phi_{3}\right|^{2}\right) \\
& -2 \nabla_{\partial_{3}}\left[\left\langle\Phi_{0}, \Phi_{2}\right\rangle-\left\langle\Phi_{1}, \Phi_{3}\right\rangle\right]+2 \nabla_{\partial_{2}}\left[\left\langle\Phi_{0}, \Phi_{3}\right\rangle+\left\langle\Phi_{1}, \Phi_{2}\right\rangle\right],
\end{aligned}
$$

$\operatorname{Re} i \nabla_{\partial / \partial q}\left\langle\Phi_{\bar{q}}, \Phi_{q^{2}}\right\rangle$

$$
\begin{aligned}
= & \frac{1}{64} \nabla_{\partial_{1}}\left(\left|\Phi_{0}\right|^{2}-\left|\Phi_{1}\right|^{2}+\left|\Phi_{2}\right|^{2}-\left|\Phi_{3}\right|^{2}\right) \\
& -2 \nabla_{\partial_{0}}\left[\left\langle\Phi_{0}, \Phi_{1}\right\rangle+\left\langle\Phi_{2}, \Phi_{3}\right\rangle\right]+2 \nabla_{\partial_{2}}\left[\left\langle\Phi_{0}, \Phi_{3}\right\rangle-\left\langle\Phi_{1}, \Phi_{2}\right\rangle\right],
\end{aligned}
$$

$\operatorname{Re} i \nabla_{\partial / \partial q}\left\langle\Phi_{\bar{q}}, \Phi_{q^{3}}\right\rangle$

$$
\begin{aligned}
= & \frac{1}{64} \nabla_{\partial_{1}}\left(\left|\Phi_{0}\right|^{2}-\left|\Phi_{1}\right|^{2}-\left|\Phi_{2}\right|^{2}+\left|\Phi_{3}\right|^{2}\right) \\
& -2 \nabla_{\partial_{0}}\left[\left\langle\Phi_{0}, \Phi_{1}\right\rangle-\left\langle\Phi_{2}, \Phi_{3}\right\rangle\right]-2 \nabla_{\partial_{3}}\left[\left\langle\Phi_{0}, \Phi_{2}\right\rangle+\left\langle\Phi_{1}, \Phi_{3}\right\rangle\right],
\end{aligned}
$$

$\operatorname{Re} j \nabla_{\partial / \partial q}\left\langle\Phi_{\bar{q}}, \Phi_{q^{1}}\right\rangle$

$$
\begin{aligned}
= & \frac{1}{64} \nabla_{\partial_{2}}\left(\left|\Phi_{0}\right|^{2}+\left|\Phi_{1}\right|^{2}-\left|\Phi_{2}\right|^{2}-\left|\Phi_{3}\right|^{2}\right) \\
& -2 \nabla_{\partial_{0}}\left[\left\langle\Phi_{0}, \Phi_{2}\right\rangle-\left\langle\Phi_{1}, \Phi_{3}\right\rangle\right]-2 \nabla_{\partial_{1}}\left[\left\langle\Phi_{0}, \Phi_{3}\right\rangle+\left\langle\Phi_{1}, \Phi_{2}\right\rangle\right],
\end{aligned}
$$


$\operatorname{Re} j \nabla_{\partial / \partial q}\left\langle\Phi_{\bar{q}}, \Phi_{q^{2}}\right\rangle$

$$
\begin{aligned}
= & \frac{1}{64} \nabla_{\partial_{2}}\left(\left|\Phi_{0}\right|^{2}-\left|\Phi_{1}\right|^{2}+\left|\Phi_{2}\right|^{2}-\left|\Phi_{3}\right|^{2}\right) \\
& +2 \nabla_{\partial_{3}}\left[\left\langle\Phi_{0}, \Phi_{1}\right\rangle+\left\langle\Phi_{2}, \Phi_{3}\right\rangle\right]-2 \nabla_{\partial_{1}}\left[\left\langle\Phi_{0}, \Phi_{3}\right\rangle-\left\langle\Phi_{1}, \Phi_{2}\right\rangle\right],
\end{aligned}
$$

$\operatorname{Re} j \nabla_{\partial / \partial q}\left\langle\Phi_{\bar{q}}, \Phi_{q^{3}}\right\rangle$

$$
\begin{aligned}
= & \frac{1}{64} \nabla_{\partial_{2}}\left(\left|\Phi_{0}\right|^{2}-\left|\Phi_{1}\right|^{2}-\left|\Phi_{2}\right|^{2}+\left|\Phi_{3}\right|^{2}\right) \\
& +2 \nabla_{\partial_{3}}\left[\left\langle\Phi_{0}, \Phi_{1}\right\rangle-\left\langle\Phi_{2}, \Phi_{3}\right\rangle\right]-2 \nabla_{\partial_{0}}\left[\left\langle\Phi_{0}, \Phi_{2}\right\rangle+\left\langle\Phi_{1}, \Phi_{3}\right\rangle\right],
\end{aligned}
$$

$\operatorname{Re} k \nabla_{\partial / \partial q}\left\langle\Phi_{\bar{q}}, \Phi_{q^{1}}\right\rangle$

$$
\begin{aligned}
= & \frac{1}{64} \nabla_{\partial_{3}}\left(\left|\Phi_{0}\right|^{2}+\left|\Phi_{1}\right|^{2}-\left|\Phi_{2}\right|^{2}-\left|\Phi_{3}\right|^{2}\right) \\
& +2 \nabla_{\partial_{1}}\left[\left\langle\Phi_{0}, \Phi_{2}\right\rangle-\left\langle\Phi_{1}, \Phi_{3}\right\rangle\right]-2 \nabla_{\partial_{0}}\left[\left\langle\Phi_{0}, \Phi_{3}\right\rangle+\left\langle\Phi_{1}, \Phi_{2}\right\rangle\right],
\end{aligned}
$$

$\operatorname{Re} k \nabla_{\partial / \partial q}\left\langle\Phi_{\bar{q}}, \Phi_{q^{2}}\right\rangle$

$$
\begin{aligned}
= & \frac{1}{64} \nabla_{\partial_{3}}\left(\left|\Phi_{0}\right|^{2}-\left|\Phi_{1}\right|^{2}+\left|\Phi_{2}\right|^{2}-\left|\Phi_{3}\right|^{2}\right) \\
& -2 \nabla_{\partial_{2}}\left[\left\langle\Phi_{0}, \Phi_{1}\right\rangle-\left\langle\Phi_{2}, \Phi_{3}\right\rangle\right]-2 \nabla_{\partial_{0}}\left[\left\langle\Phi_{0}, \Phi_{3}\right\rangle-\left\langle\Phi_{1}, \Phi_{2}\right\rangle\right],
\end{aligned}
$$

$\operatorname{Re} k \nabla_{\partial / \partial q}\left\langle\Phi_{\bar{q}}, \Phi_{q^{3}}\right\rangle$

$$
\begin{aligned}
= & \frac{1}{64} \nabla_{\partial_{3}}\left(\left|\Phi_{0}\right|^{2}-\left|\Phi_{1}\right|^{2}-\left|\Phi_{2}\right|^{2}+\left|\Phi_{3}\right|^{2}\right) \\
& -2 \nabla_{\partial_{2}}\left[\left\langle\Phi_{0}, \Phi_{1}\right\rangle-\left\langle\Phi_{2}, \Phi_{3}\right\rangle\right]+2 \nabla_{\partial_{1}}\left[\left\langle\Phi_{0}, \Phi_{2}\right\rangle+\left\langle\Phi_{1}, \Phi_{3}\right\rangle\right],
\end{aligned}
$$

$\operatorname{Re} i \nabla_{\partial / \partial q^{1}}\left\langle\Phi_{\bar{q}}, \Phi_{q}\right\rangle=\frac{1}{64} \nabla_{\partial_{1}}\left(\left|\Phi_{0}\right|^{2}+\left|\Phi_{1}\right|^{2}+\left|\Phi_{2}\right|^{2}+\left|\Phi_{3}\right|^{2}\right)$,

$\operatorname{Re} j \nabla_{\partial / \partial q^{2}}\left\langle\Phi_{\bar{q}}, \Phi_{q}\right\rangle=\frac{1}{64} \nabla_{\partial_{2}}\left(\left|\Phi_{0}\right|^{2}+\left|\Phi_{1}\right|^{2}+\left|\Phi_{2}\right|^{2}+\left|\Phi_{3}\right|^{2}\right)$,

$\operatorname{Re} k \nabla_{\partial / \partial q^{3}}\left\langle\Phi_{\bar{q}}, \Phi_{q}\right\rangle=\frac{1}{64} \nabla_{\partial_{3}}\left(\left|\Phi_{0}\right|^{2}+\left|\Phi_{1}\right|^{2}+\left|\Phi_{2}\right|^{2}+\left|\Phi_{3}\right|^{2}\right)$.

Now substituting the above expressions into (3.9) we get immediately (3.6), as required.

Definition 3.4. A function $f: \Omega \rightarrow \mathbb{H}$ is said to be antiregular in the sense of Fueter in a domain $\Omega \subset \mathbb{H}$ if $f$ is differentiable (in the usual sense) as a mapping of $\Omega$, regarded as an open set in $\mathbb{R}^{4}$, with values in $\mathbb{R}^{4}$ and

$$
D f:=\frac{1}{4}\left(\partial_{0}-i \partial_{1}-j \partial_{2}-k \partial_{3}\right)\left(f_{0}+i f_{1}+j f_{2}+k f_{3}\right)=0
$$

in $\Omega$; here $\partial_{k}:=\partial / \partial x_{k}, k=0,1,2,3$.

Remark 3.4. Let $F=F_{0}+i F_{1}+j F_{2}+k F_{3}: \Omega \rightarrow \mathbb{H}$, where $\Omega$ is an open set in $\mathbb{H}$, be a differentiable mapping (i.e. each component is differentiable as a mapping $\mathbb{R}^{4} \rightarrow \mathbb{R}$ ). Then $D F=0$ if and only if

$$
\begin{aligned}
& \partial_{0} F_{0}+\partial_{1} F_{1}+\partial_{2} F_{2}+\partial_{3} F_{3}=0, \\
& \partial_{1} F_{0}-\partial_{0} F_{1}+\partial_{2} F_{3}-\partial_{3} F_{2}=0, \\
& \partial_{2} F_{0}-\partial_{0} F_{2}-\partial_{1} F_{3}+\partial_{3} F_{1}=0, \\
& \partial_{3} F_{0}-\partial_{0} F_{3}+\partial_{1} F_{2}-\partial_{2} F_{1}=0 .
\end{aligned}
$$


Proof. This follows by straightforward computations.

Let us recall that the properties of antiregular functions are analogous to those of regular functions in the sense of Fueter (see e.g. $[6,7,13]$ ).

Theorem 3.3. Let $\Phi:\left(M^{4}, g\right) \rightarrow\left(N^{4 n}, h\right)$ be a smooth map between smooth Riemannian manifolds. Assume that $M$ is locally conformally flat. If $\Phi$ is harmonic then the function

$$
\begin{aligned}
F_{\Phi}=F_{0}+i F_{1}+j F_{2}+k F_{3}:= & \frac{1}{32}\left(\left|\Phi_{0}\right|^{2}-\left|\Phi_{1}\right|^{2}-\left|\Phi_{2}\right|^{2}-\left|\Phi_{3}\right|^{2}\right) \\
& +i\left\langle\Phi_{0}, \Phi_{1}\right\rangle+j\left\langle\Phi_{0}, \Phi_{2}\right\rangle+k\left\langle\Phi_{0}, \Phi_{3}\right\rangle
\end{aligned}
$$

is antiregular in the sense of Fueter. Moreover, if $\Phi$ is a differentiable submersion almost everywhere and $F_{\Phi}$ is antiregular in the sense of Fueter, then $\Phi$ is harmonic.

Proof. Note that the system (3.7) can be written in the following very suggestive and condensed form:

$$
\nabla_{D} F_{\Phi}=0
$$

where $D=\frac{1}{4}\left(\partial_{0}-i \partial_{1}-j \partial_{2}-k \partial_{3}\right)$ and $F_{\Phi}$ is the quaternionic-valued function defined by (3.10). Since $F_{\Phi}$ is scalar, (3.11) is equivalent to $D \cdot F_{\Phi}=0$, which proves the theorem.

The above result is rather unexpected and it emphasizes the importance of the class of regular functions in the sense of Fueter.

TheOREM 3.4. On the torus $T^{4}:=\mathbb{R}^{4} / \Lambda$, where $\Lambda$ is a lattice, consider a global linear system of coordinates $q=x_{0}+i x_{1}+j x_{2}+k x_{3}$. Then any harmonic map $\Phi: T^{4} \rightarrow\left(N^{4 n}, h\right)$ satisfies

$$
\begin{aligned}
& \left|\Phi_{0}\right|^{2}-\left|\Phi_{1}\right|^{2}-\left|\Phi_{2}\right|^{2}-\left|\Phi_{3}\right|^{2}=a, \\
& \left\langle\Phi_{0}, \Phi_{1}\right\rangle=b_{1}, \quad\left\langle\Phi_{0}, \Phi_{2}\right\rangle=b_{2}, \quad\left\langle\Phi_{0}, \Phi_{3}\right\rangle=b_{3},
\end{aligned}
$$

for suitable constants $a, b_{1}, b_{2}, b_{3} \in \mathbb{R}$.

Proof. If $\Phi$ is harmonic then $F_{\Phi}$ is antiregular in the sense of Fueter. Any antiregular function satisfies the maximum principle. Since the torus $T^{4}$ is compact, $F_{\Phi}$ has to be constant. Then, by the definition of $F_{\Phi}$, we get (3.12), as required.

Corollary 3.3. If $\Phi:\left(T^{4}, g\right) \rightarrow\left(N^{4 n}, h\right)$ is harmonic and non-constant then either $\Phi_{0}$ or $\left(\Phi_{1}, \Phi_{2}, \Phi_{3}\right)$ has no zero on $T^{4}$.

Proof. Indeed, otherwise at a point $p_{1} \in T^{4}$ where $\Phi_{0}\left(p_{1}\right)=0$, by Theorem 3.4, we would have

$$
-\left|\Phi_{1}\right|^{2}-\left|\Phi_{2}\right|^{2}-\left|\Phi_{3}\right|^{2}=a \leq 0 \quad \text { and } \quad b_{1}=b_{2}=b_{3}=0,
$$


and at a point $p_{2} \in T^{4}$ where $\left(\Phi_{1}\left(p_{2}\right), \Phi_{2}\left(p_{2}\right), \Phi_{3}\left(p_{2}\right)\right)=0$ we would get

$$
\left|\Phi_{0}\right|^{2}=a \geq 0, \quad b_{1}=b_{2}=b_{3}=0 .
$$

Then we would have

$$
a=b_{1}=b_{2}=b_{3}=0 .
$$

This means that

$$
\left\{\begin{array}{l}
\left|\Phi_{0}\right|^{2}=\left|\Phi_{1}\right|^{2}+\left|\Phi_{2}\right|^{2}+\left|\Phi_{3}\right|^{2}, \\
\left\langle\Phi_{0}, \Phi_{1}\right\rangle=\left\langle\Phi_{0}, \Phi_{2}\right\rangle=\left\langle\Phi_{0}, \Phi_{3}\right\rangle=0
\end{array}\right.
$$

at all points of $T^{4}$, with $\Phi_{i}:=\partial \Phi / \partial x_{i}, i=0,1,2,3$.

But any smooth map $\Phi:\left(T^{4}, g\right) \rightarrow\left(N^{4 n}, h\right)$ satisfying (3.13) has to be constant. Indeed, (3.13) must hold with $x_{0}$ replaced by any one of the variables $x_{1}, x_{2}, x_{3}$. Thus

$$
\left\langle\Phi_{i}, \Phi_{k}\right\rangle=0, \quad i \neq k, \quad i, k=0,1,2,3,
$$

and

$$
\left\{\begin{array}{l}
\left|\Phi_{0}\right|^{2}-\left|\Phi_{1}\right|^{2}-\left|\Phi_{2}\right|^{2}-\left|\Phi_{3}\right|^{2}=0 \\
\left|\Phi_{1}\right|^{2}-\left|\Phi_{0}\right|^{2}-\left|\Phi_{2}\right|^{2}-\left|\Phi_{3}\right|^{2}=0 \\
\left|\Phi_{2}\right|^{2}-\left|\Phi_{0}\right|^{2}-\left|\Phi_{1}\right|^{2}-\left|\Phi_{3}\right|^{2}=0 \\
\left|\Phi_{3}\right|^{2}-\left|\Phi_{0}\right|^{2}-\left|\Phi_{1}\right|^{2}-\left|\Phi_{2}\right|^{2}=0
\end{array}\right.
$$

Note that the determinant of the system (3.14) is

$$
\operatorname{det}\left(\begin{array}{cccc}
1 & -1 & -1 & -1 \\
-1 & 1 & -1 & -1 \\
-1 & -1 & 1 & -1 \\
-1 & -1 & -1 & 1
\end{array}\right) \neq 0
$$

Thus, $\Phi$ would be constant, which contradicts our assumption.

Let $S^{4}$ denote the 4-dimensional sphere.

Corollary 3.4. If $\Phi:\left(T^{4}, g\right) \rightarrow\left(S^{4}, h\right)$ is a $C^{\infty}$-map such that $\left(\Phi_{0}, \Phi_{1}\right.$, $\left.\Phi_{2}, \Phi_{3}\right) \neq 0$ on $T^{4}$ then $\operatorname{deg}(\Phi)=0$.

Pr o of. The tangent bundle $T S^{4}$ is of rank 4 , so we can complexify it. Denote by $T S_{\mathbb{C}}^{4}$ the complexification of $T S^{4}$. By the definition the bundle $\Phi^{-1}\left(T S_{\mathbb{C}}^{4}\right)$ is also a complex vector bundle (now of rank 2 ).

Denote by $c_{2}\left(\Phi^{-1}\left(T S_{\mathbb{C}}^{4}\right)\right)$ the second Chern class of $\Phi^{-1}\left(T S_{\mathbb{C}}^{4}\right)$. Since the bundle $\Phi^{-1}\left(T S_{\mathbb{C}}^{4}\right)$ is trivial we have

$$
c_{2}\left(\Phi^{-1}\left(T S_{\mathbb{C}}^{4}\right)\right)=0 .
$$

On the other hand (see $[4,11])$,

$$
c_{2}\left(T S_{\mathbb{C}}^{4}\right)=\frac{3}{4 \pi^{2}} v_{g}^{S^{4}},
$$


where $v_{g}$ is the volume form of $S^{4}$. Then we get

$$
\begin{aligned}
0 & =\int_{T^{4}} c_{2}\left(\Phi^{-1} T S_{\mathbb{C}}^{4}\right)=\int_{T^{4}} \Phi^{*}\left[c_{2}\left(T S_{\mathbb{C}}^{4}\right)\right]=\frac{3}{4 \pi^{2}} \int_{T^{4}} \Phi^{*}\left(v_{g}^{S^{4}}\right) \\
& =\frac{3}{4 \pi^{2}} \operatorname{deg}(\Phi) \operatorname{Vol}\left(S^{4}\right)=2 \operatorname{deg}(\Phi)
\end{aligned}
$$

because by the definition of the topological degree we have

$$
\int_{T^{4}} \Phi^{*}\left(v_{g}^{S^{4}}\right)=\operatorname{deg}(\Phi) \operatorname{Vol}\left(S^{4}\right) \quad \text { and } \quad \operatorname{Vol}\left(S^{4}\right)=\frac{8}{3} \pi^{2} .
$$

Thus, any $C^{\infty}$-mapping $\Phi: T^{4} \rightarrow S^{4}$ with $\left(\Phi_{0}, \Phi_{1}, \Phi_{2}, \Phi_{3}\right) \neq 0$ has degree 0 , as required.

\section{References}

[1] M. M. Berger, Sur les groupes d'holonomie homogène des variétés à connexion affine et des variétés Riemanniennes, Bull. Soc. Math. France 83 (1955), 279-330.

[2] A. L. Besse, Einstein Manifolds, Ergeb. Math. Grenzgeb. 10, Springer, Berlin, 1987.

[3] E. Bonan, Sur les G-structures de type quaternionien, Cahiers Topologie Géom. Différentielle 9 (1967), 389-461.

[4] R. Bott and L. W. Tu, Differential Forms in Algebraic Topology, Springer, New York, 1982.

[5] J. Eells and L. Lemaire, Selected Topics in Harmonic Maps, CBMS Regional Conf. Ser. in Math. 50, Amer. Math. Soc., 1983.

[6] R. Fueter, Die Funktionentheorie der Differentialgleichungen $\Delta u=0$ und $\Delta \Delta u=$ 0 mit vier reellen Variablen, Comment. Math. Helv. 7 (1935), 307-330.

[7] —, Über die analytische Darstellung der regulären Funktionen einer Quaternionenvariablen, ibid. 8 (1936), 371-378.

[8] S. Kobayashi and K. Nomizu, Foundations of Differential Geometry, Vols. I-II, Interscience, 1963.

[9] W. Królikowski, On Fueter-Hurwitz regular mappings, Dissertationes Math. 353 (1996).

[10] W. Królikowski and R. M. Porter, Quaternionic regular and biregular functions in the sense of Fueter, in: Proc. Fourth Finnish-Polish Summer School in Complex Analysis at Jyväskylä, Ber. Univ. Jyväskylä, Math. Inst. 55 (1993), 65-87.

[11] J. W. Milnor and J. D. Stasheff, Characteristic Classes, Princeton Univ. Press and Univ. of Tokyo Press, Princeton, N.J., 1974.

[12] A. J. Sommese, Quaternionic manifolds, Math. Ann. 212 (1975), 191-214.

[13] V. Souček, Holomorphicity in quaternionic analysis, in: Seminari di Geometria 1982-1983, Università di Bologna, Istituto de Geometria, Dipartimento de Matematica, 1984, 147-153. 
[14] A. Sudbery, Quaternionic analysis, Math. Proc. Cambridge Philos Soc. 85 (1979), 199-225.

Institute of Mathematics

Polish Academy of Sciences

Łódź Branch

Narutowicza 56

90-136 Łódź, Poland

Reçu par la Rédaction le 18.7.1994

Révisé le 15.2.1996 